\title{
The effectiveness of heart failure disease management: Initial findings from a comprehensive program
}

\author{
Janice L. Clarke \\ Thomas Jefferson University \\ David B. Nash \\ Thomas Jefferson University
}

Follow this and additional works at: https://jdc.jefferson.edu/healthpolicyfaculty

Part of the Health Services Research Commons

Let us know how access to this document benefits you

\section{Recommended Citation}

Clarke, Janice L. and Nash, David B., "The effectiveness of heart failure disease management: Initial findings from a comprehensive program" (2002). College of Population Health Faculty Papers. Paper 7.

https://jdc.jefferson.edu/healthpolicyfaculty/7

This Article is brought to you for free and open access by the Jefferson Digital Commons. The Jefferson Digital Commons is a service of Thomas Jefferson University's Center for Teaching and Learning (CTL). The Commons is a showcase for Jefferson books and journals, peer-reviewed scholarly publications, unique historical collections from the University archives, and teaching tools. The Jefferson Digital Commons allows researchers and interested readers anywhere in the world to learn about and keep up to date with Jefferson scholarship. This article has been accepted for inclusion in College of Population Health Faculty Papers by an authorized administrator of the Jefferson Digital Commons. For more information, please contact: JeffersonDigitalCommons@jefferson.edu. 


\title{
The Effectiveness of Heart Failure Disease Management: Initial Findings from a Comprehensive Program
}

\author{
JANICE L. CLARKE, R.N., B.B.A., and DAVID B. NASH, M.D., M.B.A.
}

\begin{abstract}
A prevalent, chronic condition among members of the mushrooming elderly population in the United States, heart failure (HF) is a logical focus for population-based disease management. Evidence supporting the premise that multidisciplinary interventions can significantly improve clinical outcomes while decreasing the cost of medical care for people with HF is steadily mounting. A growing number of controlled and observational studies focus on the effects of HF disease management on re-admission rates, length of stay, and improvement in appropriate diagnostic testing and prescribing. This paper describes a large-scale, comprehensive HF program and reports on clinical quality, utilization, and financial outcomes observed after 1 year. The preliminary findings strengthen the case for comprehensive HF disease management as an effective means for improving clinical outcomes and reducing total medical costs for large patient populations.
\end{abstract}

\section{INTRODUCTION}

A DEVASTATING DISEASE in terms of its prevalence, morbidity and mortality, and financial costs, heart failure (HF) ranks high on the list of chronic conditions burdening the U.S. health care system today. Recent statistics show that HF affects an estimated 5 million Americans, with approximately 400,000 new cases diagnosed each year. ${ }^{1}$ Jolting as these statistics are, they are likely an underestimation of the actual incidence of HF. In one retrospective review of 225 randomly selected patients, investigators concluded that many patients admitted with shortness of breath and low left ventricular ejection fraction had HF but are not diagnosed. ${ }^{2}$

A patient's prospects for survival following a diagnosis of HF are bleaker than those for most forms of cancer. HF carries an overall mortality rate of $50 \%$ within 5 years of diagnosis. Croft et al. ${ }^{3}$ reported a 6-year mortality rate secondary to $\mathrm{HF}$ of $84 \%$ in men and $77 \%$ in women. Despite continued advances in medical treatment for the disease, the mortality and morbidity attributed to HF have continued to rise. Between 1979 and 1997, HF deaths increased by $145 \%$ and HF hospitalizations rose by $155.2 \%$.

When viewed in the context of an aging U.S. population, the implications of the preceding take on added dimension. As 75 million "babyboomers" age, the number of Americans over the age of 65 is expected to double over the next 30 years. ${ }^{5}$ The incidence of HF increases with age: In people over the age of 65 it approaches $1 \%$, and in the $70-79$ age group it increases to $4 \%$. By the year 2007, the prevalence of HF is

Office of Health Policy and Clinical Outcomes, Thomas Jefferson University Hospital, Philadelphia, Pennsylvania. 
expected to reach 10 million cases in the United States alone. ${ }^{6}$

According to Medicare program (HCFA/ CMS) data, expenditures for HF in 1991 were higher than the combined expenditures for the five most common diagnosis-related groups for cancer, and higher than those for myocardial infarction. ${ }^{7}$ In dollars, HF management, including hospitalization costs (inpatient and pharmacy) and outpatient visits, approached $\$ 38$ billion. By 1996, HF accounted for $2.8 \%$ of all hospital discharges and $22 \%$ of all discharges for cardiovascular disease in the United States. "Based on an increase in the number of patients with heart failure since 1991, the total cost for HF management for 1999 is estimated to approach $\$ 56$ billion." 6 Data suggest that half of the $15-30 \%$ HF related readmissions (within 90 days) are preventable.

\section{Barriers to effective outpatient management}

Medical management after the onset of HF is challenging for both physicians and patients. For the primary care physician (PCP), each new clinical trial and each new or revised guideline increases the complexity of the care and treatment of the patient with HF, particularly the patient with a co-existing chronic condition. A majority of patients with HF have one or more comorbid conditions. Diabetes, a complex disease in its own right, is a common co-existing condition, as are hypertension, hypercholesterolemia, and depression.

National guidelines for the medical management of patients with HF, published by the Agency for Healthcare Research and Quality (AHRQ) and the American College of Cardiology (ACC)/American Heart Association (AHA), are readily available from a variety of sources, including medical professional organizations, government agencies, managed care organizations, and other health care insurers. However, the administrative data [for example, Health Plan Employer Data and Information Set (HEDIS $^{\circledR}$; National Committee for Quality Assurance) data] reported by health plans show persistent and disturbing variances between recommended treatment and standard practice. Physician adherence to the established guidelines for the use of angiotensin convert- ing enzyme (ACE) inhibitors has been the focus of numerous studies. While there is plentiful evidence that ACE inhibitors reduce disability, improve functional capacity, and prolong life in patients with HF, studies show that physicians continue to underutilize these drugs. In one study, only $73 \%$ of the patients most likely to benefit from and tolerate such therapy were prescribed ACE inhibitors at discharge from the hospital. ${ }^{8}$

Barriers to effective treatment of HF are not limited to physician issues. Cardiovascular disease often arises from unhealthy lifestyle patterns such as smoking, poor diet, or inactivity. Patients with HF often find it difficult to make necessary changes in behavior and lifestyle. Chin and Goldman 9 found that some admissions for HF could be attributed to patient inability to adhere to dietary (salt) restrictions or medication regimens. Psychosocial factors such as depression, hostility, and the social isolation often associated with age and infirmity have all been shown to contribute independently to mortality and morbidity in patients with cardiovascular disease-and anger and hostility are strongly associated with the development of HF.

Of particular interest are findings suggesting that as many as $15 \%$ of hospital readmissions for HF are related to underutilization of prescribed medication. ${ }^{10}$ In his discussion of the economic burden of $\mathrm{HF}, \mathrm{O}^{\prime}$ Connell ${ }^{6}$ estimates that "if pharmacologic and behavioral approaches were combined, the five-year mortality rate could be reduced by as much as 50 percent."

\section{Disease management approaches to HF}

Successful outpatient management models have been described in the literature, each employing a systematic approach to HF care and each resulting in improved quality as measured by decreased utilization of health care resources or by increased utilization of appropriate medications. Multiple approaches including outpatient HF clinics, predischarge team planning, off-site case management with home visits, and Internet-based interventions have been developed and researched. Positive findings consistently include reduction in adverse clinical outcomes (e.g., readmissions) and 
costs with corresponding improvement in medication use and quality-of-life (QOL) indicators. ${ }^{11-15}$ In a critical review of the evidence for $\mathrm{HF}$ disease management programs prior to 1999, Rich ${ }^{16}$ catalogued 10 observational studies that suggested an association between multidisciplinary approaches and reduced inpatient admissions and length of stay (LOS), improved QOL, and lower overall cost of care. He also reviewed six randomized studies that effectively reduced inpatient utilization, improved functional capacity, and enhanced patient knowledge and compliance with treatment regimens. The long-term impact and cost-effectiveness of these models have yet to be determined.

Evidence of value of programs focused on developing self-management skills continues to mount. A recent AHRQ study of self-management programs at several university-based centers concluded that such programs improve health and reduce use of health care resources by people with chronic diseases. ${ }^{17}$ In evaluating HF disease management programs, brief interventions (requiring 80-120 min of physician and other health care provider time) have been shown to be effective for patients who are highly motivated, have less comorbidity, are better educated, and have more social support. For patients who need more motivation, support, or skills training than a brief intervention can provide, intensive interventions have been shown to produce a greater effect than brief interventions. ${ }^{18}$

\section{PROGRAM DESCRIPTION}

American Healthways Corporation (AMHC) provides the Cardiac Healthways ${ }^{\mathrm{SM}} \mathrm{HF}$ Program described herein to members of a large (over 800,000 members) midwestern U.S. commercial health plan. Holistic in approach, the program is designed to coordinate all health care needs of participants, not just those related to the managed disease. Stated program goals include promoting the physician-patient relationship; supporting the physician with patient management through regular health assessments, symptom monitoring, and ongoing patient education; improving the health status of the population; and, as a result, reducing the cost of medical care. The program is grounded in nationally recognized, evidence-based guidelines for medical management and treatment of heart failure, including the ACC/ AHA Guidelines for the Evaluation and Management of Heart Failure; the National Cholesterol Education Program Guidelines (ATP III); the Joint National Committee for Treatment of High Blood Pressure (JNC VI); and the ACC/AHA Guidelines for Preventing Heart Attack and Death in Patients with Atherosclerotic Cardiovascular Disease: 2001 Update.

\section{Education and support for PCPs}

Cooperation and communication with the PCP are essential to the success of a disease management program developed around a pri-

Table 1. Focused Interventions for PCPs

Physician intervention

Frequency

Introductory letter

Cardiac care guidelines

Sample participant "welcome kit"

Provider service manager visit

Single topic sheet: beta-blocker indications and dosing

Single topic sheet: ACE inhibitor indications and dosing

Participant medication list

Computer-generated alerts/reminders

Patients not on ACE inhibitors or beta-blockers

Home-monitoring alerts re weight, blood pressure, pulse

Cardiac-specific newsletter

Toll-free hotline

Flow sheet for documenting compliance with guidelines

Satisfaction survey
Initially

Annually, as updated

Initially

Minimum once

Semiannually

Semiannually

Daily, as needed

Quarterly

Ongoing

Initial and annually

Minimum annually 
mary care "gatekeeper." The PCP is brought into the loop at the time of a patient's enrollment, and frequent contact is made throughout the patient's participation in the program (Table 1). Program staff sends an introductory letter along with a synopsis of the most recent ACC / AHA cardiac care guidelines to the PCP. Medication list updates, including both pharmaceutical claims data and patient self-reported information, are sent periodically to the PCP for all her/his participating patients. Software-generated reports are sent to alert physicians of HF patients who may be appropriate for certain treatments, such as ACE inhibitors or beta-blockers, and whose pharmacy records indicate that these medications are not prescribed.

A group of specially trained registered nurses, assigned to physician territories, visit offices to orient PCPs and their office staffs to the HF program. They provide education, offer encouragement and tools to assist the PCP in adhering to standards of care (e.g., indications for prescribing ACE inhibitors), and establish collaborative relationships to facilitate follow-up visits.

\section{Focused interventions for program participants}

Program participant interventions and associated frequencies are listed in Table 2. The key participant intervention is the "Care Call." Care Calls, regularly scheduled telephone contacts initiated by the program's nursing staff, serve as a basis for conducting health assess- ments as well as providing disease-specific education, advocacy, and provider treatment plan support on an ongoing basis. Participant responses to the assessments direct the nurses to educational points for discussion with the participant. At each follow-up phone call, nurses use a "follow-up assessment" script designed to quickly identify any decompensating symptoms. In the event of urgent symptoms, the nurses reinforce self-management by assuring that patients make contact with their PCPs. All HF participants meet criteria for the highest level (Level 4) in the program's proprietary population stratification model, and, accordingly, they are referred to nurses with the highest level of training for Care Calls. On the initial call, the nurse conducts a thorough HF assessment to further stratify the patient, which, in turn, drives the scheduled Care Call frequency. Care Calls range from weekly to every 6 weeks for HF members.

The nurse conducts a thorough medication review on the initial call, cataloguing all medications the participant is taking along with dosages and frequencies, compliance, medication knowledge, and attitude toward taking medications. From this discussion, the nurse is directed to education points on medications and suggested questions for the participant to ask his/her physician (i.e., "Would a betablocker be a good medication for me to be on?" "Should I be taking an ACE inhibitor?"). During follow-up calls, the nurse asks about any changes in medications.

\begin{tabular}{|ll|}
\hline \multicolumn{1}{|c|}{ Table 2. Focused Interventions fOr HF Program Participants } \\
\hline Participant interventions & \multicolumn{1}{c|}{ Frequency } \\
\hline Letter of introduction and welcome kit & On identification \\
Standards of care mailings & Quarterly \\
Health education mailings & As requested by member or PCP \\
Cardiac-specific newsletters & Quarterly \\
Care Calls & Once \\
Initial welcome call & Annually \\
Assessments (e.g., HF, medication, health status, & \\
depression risk screen, QOL) & According to protocol \\
Scheduled follow-up calls and re-assessment & According to protocol \\
In-home telemonitoring (weight, blood pressure, pulse) & Ongoing \\
Self-Care Goal Agreement & Ongoing \\
CHF toll-free hotline & Ongoing \\
CHF website & Minimum annually \\
Satisfaction survey & \\
\hline
\end{tabular}


Whenever the nurse perceives a participant knowledge deficit, he/she supplements the discussion with a mailed single topic information sheet. Examples include information specific to medications such as ACE inhibitors, beta-blockers, aspirin, or statins; nutrition (low sodium diet); or congestive HF (CHF) (e.g., "What is CHF?"). All information from calls is entered in the HF participant's electronic medical record.

Another important element in the Care Call is the Self-Care Goal Agreement. A product of the nurse-participant telephone discussions, the agreement documents the participant's identified self-care goals. The goals are directed toward behavior modification that supports both the physician's treatment plan and the participant's objectives.

By means of these regular interactions with program participants, nurses are able to identify those patients most likely to benefit from in-home monitoring. After consultation with a patient's PCP, the nurse may arrange for electronic monitoring of the patient's weight, heart rate, and blood pressure. Patient selection and the duration of the in-home monitoring are determined by protocol.

\section{SUBJECTS AND METHODS}

The program is provided on an engagement basis to health plan members (i.e., all Com- mercial and Medicare + Choice members identified as having HF are automatically included in the program unless they exercise their right to "opt-out" by choosing not to participate). Identification is made on the basis of a minimum of one HF inpatient claim/encounter or two HF outpatient claims/encounters within a 12-month period with matching primary or secondary ICD-9 diagnosis codes listed in Table 3. Types of claims include professional, facility, and ancillary (inpatient, outpatient, home health, pharmacy, durable medical equipment, and any other ancillary) charges. Once identified, members and their PCPs are welcomed to the program via introductory letters, welcome kits, and Standards of Care notices. An electronic database equipped with proprietary software enables program staff to track participants' medical claims, drug prescriptions, nature and frequency of laboratory tests, and, in most cases, laboratory test results.

\section{INITIAL RESULTS}

Table 4 provides summary demographic data for program participants. Participant characteristics were well balanced with respect to age and gender for the baseline and report periods. Change in male-female ratio over the report period was marginally significant $(p<$ 0.05). More than one-third of participants had

Table 3. Identification Scheme for HF Program

\begin{tabular}{ll}
\hline ICD-9 code & \multicolumn{1}{c}{ Description } \\
\hline 398.91 & Other rheumatic heart disease, rheumatic HF (congestive) \\
402.01 & Hypertensive heart disease, malignant, with CHF \\
402.11 & Hypertensive heart disease, benign, with CHF \\
402.91 & Hypertensive heart disease, unspecified, with CHF \\
404.01 & Hypertensive heart and renal disease, malignant, with CHF \\
404.02 & Hypertensive heart and renal disease, malignant, with CHF and renal failure \\
404.11 & Hypertensive heart and renal disease, benign, with CHF \\
404.12 & Hypertensive heart and renal disease, benign, with CHF and renal failure \\
404.91 & Hypertensive heart and renal disease, unspecified, with CHF \\
404.93 & Hypertensive heart and renal disease, unspecified, with CHF and renal failure \\
425.0 & Cardiomyopathy \\
428.0 & HF, CHF \\
428.1 & HF, left HF \\
428.9 & HF, unspecified \\
\hline
\end{tabular}

Health plan members were identified for inclusion in the program based on at least one inpatient claim/encounter or two outpatient claims/encounters within a 12-month period with matching ICD-9 diagnostic codes listed. 


\begin{tabular}{|c|c|c|}
\hline & Baseline & Year 1 \\
\hline Total participants & $1,637(100 \%)$ & $1,973(100 \%)$ \\
\hline \multicolumn{3}{|l|}{ Age groupings } \\
\hline $19-34$ & $17(1.0 \%)$ & $22(1.1 \%)$ \\
\hline $35-50$ & $118(7.2 \%)$ & $102(5.2 \%)$ \\
\hline $51-65$ & $326(19.9 \%)$ & $425(21.5 \%)$ \\
\hline $66-80$ & $643(39.2 \%)$ & $694(35.2 \%)$ \\
\hline $81+$ & $533(32.6 \%)$ & $730(37.0 \%)$ \\
\hline \multicolumn{3}{|l|}{ Gender } \\
\hline Female & $914(55.8 \%)$ & $1,032(52.3 \%)$ \\
\hline Male & $723(44.2 \%)$ & $941(47.7 \%)$ \\
\hline \multicolumn{3}{|l|}{ Comorbidities } \\
\hline CHF only & $1,070(65.4 \%)$ & $1,251(63.4 \%)$ \\
\hline CHF with diabetes & $567(34.6 \%)$ & $722(36.6 \%)$ \\
\hline Continuously enrolled & $1,218(74.4 \%)$ & $1,456(73.8 \%)$ \\
\hline
\end{tabular}

diabetes as a comorbid condition. Of health plan members identified with HF and enrolled in the program, 641 opted out. There were no apparent characteristics that distinguished this group from the participant group on analysis. Approximately $75 \%$ of participants were continuously enrolled in the program. One hundred twenty deaths (approximately 6\%) were identified among program participants during the first year (June 1, 2000-May 31, 2001), an appreciably lower rate than expected in the general population (i.e., overall death rate from HF in the United States in 1999 was $18.8 \%$ ).

Outcome variables for the first year of the program (June 1, 2000-May 31, 2001 with 6month run-out) were compared with participant historical data in the year prior to engagement in the program (Baseline; January 1, 1999-December 31, 1999 with 6-month runout).

\section{CLINICAL PROCESS OUTCOMES}

Three clinical processes were examined for indications of program effectiveness. The data included patients with systolic as well as diastolic dysfunction.

\section{Appropriate use of ACE inhibitors}

For the baseline and report period, the number and percentage of all program participants on an ACE inhibitor were calculated. Those taking an angiotensin receptor blocker or a combination of hydralazine and nitrates were included in the numerator.

\section{Appropriate use of beta-blockers}

For the baseline and report period, the number and percentage of program participants on a beta-blocker following diagnosis of myocardial infarction were calculated.

\section{Appropriate low-density lipoprotein cholesterol (LDL-C) screening}

For the baseline and report periods, the number and percentage of all program participants with administrative evidence of having a lipid profile screening were calculated. LDL-C profiles are presumed to be due 12 months following a preceding screening. Table 5 shows statistically significant $(p<0.001)$ improvement in appropriate utilization of ACE inhibitors and LDL-C testing for program participants during the first year of enrollment. While a $16.4 \%$ improvement was observed in betablocker utilization following myocardial in-

Table 5. Changes in Clinical Quality Process Measures for Members Enrolled in the HF Program

\begin{tabular}{|c|c|c|c|c|c|c|c|}
\hline \multirow[b]{2}{*}{ Clinical measure } & \multicolumn{3}{|c|}{ Baseline $(1,647$ members $)$} & \multicolumn{3}{|c|}{ Year 1 (1,973 members) } & \multirow[b]{2}{*}{$\chi^{2}(\mathrm{p})$} \\
\hline & $N$ & $D$ & $\%$ & $N$ & $D$ & $\%$ & \\
\hline LDL & 320 & 1,637 & $19.5 \%$ & 741 & 1,973 & $37.6 \%$ & $139.82(<0.001)^{1}$ \\
\hline $\mathrm{ACE}^{2}$ & 702 & 1,389 & $50.5 \%$ & 1,092 & 1,707 & $64.0 \%$ & $59.95(<0.001)$ \\
\hline Post-myocardial infarction & 30 & 70 & $42.9 \%$ & 32 & 54 & $59.3 \%$ & 3.28 (n.s.) \\
\hline
\end{tabular}

The percentages with each clinical measure were calculated from the given numerator $(\mathrm{N})$ and denominator $(\mathrm{D})$. n.s., not significant.

${ }^{1}$ Critical value at $p=0.001: 10.827$.

${ }^{2}$ Excludes members with history of renal failure and members with medication allergy to ACE inhibitors who are not taking an angiotensin receptor blocker or a combination of hydralazine and nitrates. 


\begin{tabular}{|c|c|c|c|}
\hline & Baseline $(1,637$ members $)$ & Year 1 (1,973 members) & $\%$ change \\
\hline Admissions per 1,000 & $\begin{array}{c}1,409.5 \\
(\text { total }=2,008)\end{array}$ & $\begin{array}{c}1,149.2 \\
\text { (total }=1,949)\end{array}$ & $-18.5 \%$ \\
\hline Days per 1,000 & $9,416.2$ & $7,324.9$ & $-22.2 \%$ \\
\hline Average LOS & 6.7 days & 6.4 days & $-4.4 \%$ \\
\hline ER admissions per 1,000 & $\begin{array}{c}1,239.6 \\
(\text { total }=1,766)\end{array}$ & $\begin{array}{c}935.1 \\
\text { (total }=1,586)\end{array}$ & $-24.6 \%$ \\
\hline
\end{tabular}

farction, significance was borderline at the $p<$ 0.05 level. In all likelihood, the small numbers account for this.

\section{OTHER OUTCOMES}

A comparison of hospital utilization patterns for the two periods yielded additional positive findings. Total admissions decreased, and, as shown in Table 6, reductions were realized in the number of inpatient admissions per thousand $(18.5 \%)$, days per thousand $(22.2 \%)$, and average LOS $(4.4 \%)$. Visits to the emergency room (ER), costly in both human and financial terms, also decreased in the first year. ER admissions per thousand dropped by $24.6 \%$. A preliminary examination of readmission rates showed a decline in the number of readmissions within 30 (18.8\%) and 60 (19.2\%) days of discharge with a primary diagnosis of $\mathrm{CHF}$ (ICD-9 code 428.0). Adjusting for the difference in member months, the percentage reductions are even greater $(-31.7 \%$ and $-32.2 \%$, respectively).
A cost adjustment was necessary in order to make valid financial comparisons between the two periods. An "inflation factor," reflecting the increase in total medical costs over the period, was calculated using medical claims for the "nondisease" population. The nondisease population, defined as all health plan members without any indication of either HF or diabetes, experienced an $18.2579 \%$ increase in medical costs. This estimate of the overall increase in costs was used as a multiplier in adjusting baseline figures for financial comparisons. A comparison of inflation-adjusted financial data from the year prior to implementing the program and the first year of the program showed total medical dollars per member per month (PM/PM) decreasing by $28 \%$, with the expected increase in pharmacy costs being offset by the sharper reduction in medical spending for urgent and inpatient care (Table 7).

Finally, summary data from a satisfaction survey of a random sample of 103 (approximately $6 \%$ ) program participants yielded findings consistent with the preceding positive outcomes. Of the respondents, $89.4 \%$ rated their

\begin{tabular}{|c|c|c|c|c|}
\hline & \multicolumn{2}{|c|}{$\begin{array}{c}\text { Baseline }(1 / 1 / 99-12 / 31 / 99) \\
(1,637 \text { members })\end{array}$} & \multicolumn{2}{|c|}{$\begin{array}{c}\text { Year } 1(6 / 1 / 00-5 / 31 / 01 \\
(1,973 \text { members })\end{array}$} \\
\hline \multirow[t]{2}{*}{ Member months } & \multicolumn{2}{|c|}{17,096} & \multicolumn{2}{|c|}{20,352} \\
\hline & & Adjusted base ${ }^{1}$ & & $\%$ change \\
\hline Medical dollars PM/PM & 1,060 & $1,253.74$ & 870 & $-30.6 \%$ \\
\hline Pharmacy dollars PM/PM & 66 & 77.71 & 89 & $+14.5 \%$ \\
\hline Total dollars PM/PM & 1,126 & $1,331.45$ & 959 & $-28.0 \%$ \\
\hline Total medical expenditures & $\$ 19,248,198$ & $\$ 22,762,514$ & $\$ 19,519$ & \\
\hline
\end{tabular}

${ }^{1}$ Cost-adjusted for inflation in medical costs: baseline multiplier $18.2579 \%$. 
overall satisfaction with the program from "good" to "excellent."

\section{DISCUSSION}

The observed increases in pharmacy and ACE inhibitor utilization are indications that targeted program interventions had the desired effect on the behavior of both patients and their physicians. Summary statistics for in-office education with provider offices for the first year revealed the intensity of program communication efforts: 317 office/clinic visits, 258 physician interactions, and 325 clinical staff interactions. One question to be addressed in the future is why, with the program's emphasis on proper use of and dosing with ACE inhibitors, the utilization of ACE inhibitors in this population is less than the utilization reported in studies of smaller populations. ${ }^{11,12}$

The phenomenon of "regression to the mean" is understood to be a factor in explaining decreases in medical costs for HF populations, particularly those identified on the basis of a hospitalization in the baseline year. To minimize the effect of this potential bias, the program population was identified via outpatient as well as inpatient data, and the analyses include members with and without hospitalizations for both the baseline and the report period. Medical expenses for health plan members without evidence of HF or diabetes rose by $18.25 \%$ during the report period, supporting the premise that the positive financial outcomes observed were due to the intervention rather than regression to the mean.

Using cost-adjusted medical expenditures for the baseline, estimated total cost savings for program participants in the first year is $\$ 6,359,712$. The average first year savings for a HF program are $10 \%$ beyond the cost of the program. While the costs of providing this program are not available for publication, the financial outcomes suggest that the program was cost-effective.

\section{CONCLUSION}

HF is aptly described as an "emerging epidemic" in the Medicare population. Prospects for survival for older adults with HF in the United States are dismal. Growing numbers of adults are at increased risk for developing HF due to hypertension, diabetes, and/or myocardial infarction. Evidence of the effectiveness of HF disease management has been reported in a number of small population studies. This observational study extends our understanding of the role of HF disease management in controlling or retarding the progression of HF, improving participants' health status, and decreasing overall medical costs in a large population. While it is recognized that it may take several years to fully determine its effectiveness, the initial outcomes of this HF disease management program are uniformly positive. Analyses of clinical processes and a spectrum of other variables, including medical utilization, financial data, and participant satisfaction, suggest that the program's multiple physician- and patient-focused interventions are associated with significant improvement in outpatient medical management, reduced inpatient utilization, and decreased medical costs for participants.

The evidence for HF disease management is growing, but, if the staggering clinical and economic effects of the disease are to be contained, the health care industry must continue to develop and refine strategies for controlling the progression of HF in diagnosed patients and for preventing the onset of HF. Proactive steps must be taken to control the condition prior to the onset or early in the course of progressive left ventricular dysfunction. Predictive models to identify patients likely to develop HF and early interventions to head off adverse clinical events are already being studied. Incorporation of primary prevention strategies, such as treatment of hypertension and hyperlipidemia and intensive education for physicians and patients alike, holds promise for improving or reversing myocardial dysfunction. A multifaceted program such as the one described could become even more effective by broadening its scope to include prevention.

\section{ACKNOWLEDGMENTS}

This study was supported by an unrestricted grant from AMHC. The authors have no fi- 
nancial interest or stake in AMHC. The cooperation of AMHC staff (Thad Perry, Ph.D., Vice President of Informatics; Brent Hamar, D.D.S., M.P.H., Manager of Informatics; Pat Hamilton, Director of Product Development and Management; and Matthew McGinnis, Manager of Contract Performance) and the staff of Thomas Jefferson University's Office of Health Policy and Clinical Outcomes (Albert Crawford, Ph.D.; Christine Weston, M.S. Ed., Ph.D.; and Christine Hartmann, M.S.; Project Directors) is greatly appreciated.

\section{REFERENCES}

1. National Heart, Lung, and Blood Institute, National Institutes of Health. Congestive heart failure in the United States: a new epidemic. Bethesda, MD: National Institutes of Health, 1996.

2. Akosah KO, Moncher K, Schaper A, Havlik P, Devine $\mathrm{S}$. Chronic heart failure in the community: missed diagnosis and missed opportunities. J Card Fail 2001; 7:232-238.

3. Croft JB, Giles WH, Pollard RA, Keenan NL, Casper ML, Anda RF. Heart failure survival among older adults in the United States: a poor prognosis for an emerging epidemic in the Medicare population. Arch Intern Med 1999;159:505-510.

4. American Heart Association. 2002 heart and stroke statistical update. Dallas: American Heart Association, 2002:19.

5. American College of Cardiology. ACC/AHA guidelines for the evaluation and management of chronic heart failure in the adult: a report of the American College of Cardiology/American Heart Association Task Force on Practice Guidelines. Available at http://www.acc.org. Accessed April 17, 2002.

6. O'Connell JB. The economic burden of heart failure. Clin Cardiol 2000;23(Suppl 3):III-6-III-10.

7. O'Connell JB, Bristow MR. Economic impact of heart failure in the United States: time for a different approach. J Heart Lung Transplant 1994;13(Suppl): S107-S112.

8. Heart failure treatment with angiotensin-converting enzyme inhibitors in hospitalized Medicare patients in 10 large states. The Large State Peer Review Orga- nization Consortium. Arch Intern Med 1997;157: 1103-1108.

9. Chin MH, Goldman L. Factors contributing to the hospitalization of patients with congestive heart failure. Am J Public Health 1997;87:643-648.

10. Vinson JM, Rich MW, Sperry JC, Shah AS, McNamara T. Early readmission of elderly patients with congestive heart failure. J Am Geriatr Soc 1990;38:1290-1295.

11. Whellan DJ, Gaulden L, Gattis WA, et al. The benefit of implementing a heart failure disease management program. Arch Intern Med 2001;161:2223-2228.

12. Kasper EK, Gerstenblith G, Hefter G, et al. A randomized trial of the efficacy of multidisciplinary care in heart failure outpatients at high risk of hospital readmission. J Am Coll Cardiol 2002;39:471-480.

13. Krumholtz HM, Amatruda J, Smith GL, et al. A randomized trial of an education and support intervention to prevent readmission of patients with heart failure. J Am Coll Cardiol 2002;39:83-89.

14. Reigel B, Carlson B, Kopp Z, LePetri B, Glaser D, Unger A. Effect of a standardized nurse case-management telephone intervention on resource use in patients with chronic heart failure. Arch Intern Med 2002;162:705-712.

15. Holst DP, Kaye D, Richardson M, et al. Improved outcomes from a comprehensive management system for heart failure. Eur J Heart Fail 2001;3:619-625.

16. Rich MW. Heart failure disease management: a critical review. J Card Fail 1999;5:64-75.

17. Agency for Healthcare Research and Quality. Selfmanagement programs found to improve health and reduce use of health care by people with chronic diseases. Rockville, MD: Agency for Healthcare Research and Quality, 2002;258:1-2.

18. Center for the Advancement of Health. Health behavior change in managed care: selected evidence for behavioral approaches to chronic disease management in clinical settings. Available at http://www. cfah.org. Accessed June 4, 2002.

Address reprint requests to: David B. Nash, M.D., M.B.A. Office of Health Policy and Clinical Outcomes Thomas Jefferson University Hospital 1015 Walnut Street, Suite 115 Philadelphia, PA 19107

E-mail: David.Nash@mail.tju.edu 